PROCEEDINGS OF THE AMERICAN MATHEMATICAL SOCIETY

Volume 125, Number 3, March 1997, Pages 779-784

S 0002-9939(97)03590-9

\title{
NONCOMMUTATIVE $H^{2}$ SPACES
}

\author{
MICHAEL MARSALLI
}

(Communicated by Palle E. T. Jorgensen)

\begin{abstract}
Let $\mathcal{M}$ be a von Neumann algebra with a faithful, finite, normal tracial state $\tau$, and let $\mathcal{A}$ be a finite, maximal subdiagonal algebra of $\mathcal{M}$. Let $H^{2}$ be the closure of $\mathcal{A}$ in the noncommutative Lebesgue space $L^{2}(\mathcal{M}, \tau)$. Then $H^{2}$ possesses several of the properties of the classical Hardy space on the circle, including a commutant lifting theorem, some results on Toeplitz operators, an $H^{1}$ factorization theorem, Nehari's Theorem, and harmonic conjugates which are $L^{2}$ bounded.
\end{abstract}

\section{INTRODUCTION}

In [1] Arveson introduced the concept of a subdiagonal algebra in order to unify the analysis of several broad classes of nonselfadjoint operator algebras. Arveson drew a close analogy between a subdiagonal algebra and the classical Hardy space $H^{\infty}$, the boundary values of the bounded analytic functions on the disk. Roughly, a subdiagonal algebra $\mathcal{A}$ stands in relation to its von Neumann algebra $\mathcal{M}$ as $H^{\infty}$ stands in relation to the Lebesgue space $L^{\infty}$ of the unit circle. Subsequently, several authors studied the invariant subspaces of $\mathcal{A}$ acting on the noncommutative Lebesgue space $L^{p}$ [6], [8], [10]. There has also been considerable investigation of analytic crossed products, which are a type of subdiagonal algebra introduced by McAsey, Muhly and Saito, including their invariant subspace structure [8], [10], maximality among weak ${ }^{*}$ closed subalgebras of $\mathcal{M}[8]$, associated Toeplitz operators [11] and Hankel operators [5]. We shall study the closure of $\mathcal{A}$ in $L^{2}$ as an analogue of the classical Hardy space $H^{2}$, and so obtain analogues of several classical results including a commutant lifting theorem, some results on Toeplitz operators, an $H^{1}$ factorization theorem, Nehari's Theorem on the norm of a Hankel operator, and the existence and $L^{2}$ boundedness of the harmonic conjugate.

Let $\mathcal{M}$ be a von Neumann algebra with a faithful, normal finite tracial state $\tau$. For $1 \leq p<\infty$, let $L^{p}=L^{p}(\mathcal{M}, \tau)$ denote the noncommutative Lebesgue space which is associated with $\mathcal{M}$ and $\tau$ (cf. [3], [9], [12]). For $t \in \mathcal{M}, x \in L^{2}$, let $L_{t}(x)=t x$ and $R_{t}(x)=x t$. Then $\mathcal{L}=\left\{L_{t}: t \in \mathcal{M}\right\}$ and $\mathcal{R}=\left\{R_{t}: t \in \mathcal{M}\right\}$ are von Neumann algebras on $L^{2}$ which are each other's commutants. Furthermore, the map $t \rightarrow L_{t}$ (resp. $t \rightarrow R_{t}$ ) is a normal, *-isomorphism (resp. *-anti-isomorphism) of $\mathcal{M}$ onto $\mathcal{L}$ (resp. $\mathcal{R}$ ), and the identity 1 is a cyclic and separating vector for $\mathcal{L}$ and $\mathcal{R}$. The map $x \rightarrow x^{*}$ on $\mathcal{M}$ extends to a conjugate linear isometry on $L^{p}$. As is customary, we identify $\mathcal{M}$ with $L^{\infty}$ while the ultraweak topology on $\mathcal{M}$ will

Received by the editors July 10, 1995 and, in revised form, July 27, 1995.

1991 Mathematics Subject Classification. Primary 47D15, 46L50.

(C)1997 American Mathematical Society 
be identified with the weak ${ }^{*}$ topology on $L^{\infty}$ regarded as the dual of $L^{1}$. We now introduce $\mathcal{A}$, the noncommutative analogue of $H^{\infty}$ (cf. [1], [8]).

Definition. Let $\mathcal{A}$ be a $\mathrm{w}^{*}$-closed unital subalgebra of $\mathcal{M}$, and let $\Phi$ be a faithful, normal expectation from $\mathcal{M}$ onto the diagonal $\mathcal{D}=\mathcal{A} \cap \mathcal{A}^{*}$. Then $\mathcal{A}$ is a finite, maximal subdiagonal subalgebra of $\mathcal{M}$ with respect to $\Phi$ if:

(1) $\mathcal{A}+\mathcal{A}^{*}$ is $\mathrm{w}^{*}$-dense in $\mathcal{M}$,

(2) $\Phi(a b)=\Phi(a) \Phi(b)$ for all $a, b \in \mathcal{A}$,

(3) $\mathcal{A}$ is maximal among those subalgebras satisfying (1) and (2), and

(4) $\tau \circ \Phi=\tau$.

For $\mathcal{S} \subset L^{p}, 1 \leq p<\infty$, let $[\mathcal{S}]_{p}$ denote the closure of $\mathcal{S}$ in $L^{p}$. Let $H^{p}=[\mathcal{A}]_{p}$, $H_{0}^{p}=[\{x \in \mathcal{A}: \Phi(x)=0\}]_{p}$, and $P$ be the orthogonal projection of $L^{2}$ onto $H^{2}$. Then $\Phi$ extends to the orthogonal projection of $L^{2}$ onto $[\mathcal{D}]_{2}$ and $L^{2}=H^{2} \oplus\left(H_{0}^{2}\right)^{*}=$ $H_{0}^{2} \oplus[\mathcal{D}]_{2} \oplus\left(H_{0}^{2}\right)^{*}$ by [8, Proposition 1.1]. For $t \in \mathcal{M}$ we define the (left) Toeplitz operator with symbol $t$ by $T_{t}=P L_{t} P$. We define the (left) Hankel operator with symbol $t$ by $H_{t}=(1-P) L_{t} P$.

\section{A commutant Lifting TheOREM}

Let $\mathcal{T}$ denote the algebra $\left\{L_{a}: a \in \mathcal{A}\right\}$ considered as an algebra of operators on $H^{2}$. In this section we identify $\mathcal{T}^{\prime}$, the operators on $H^{2}$ that commute with $\mathcal{T}$. In particular, we show that every element of $\mathcal{T}^{\prime}$ lifts to an element of $\mathcal{R}$. Our result is analogous to Yoshino's Theorem [13], which describes the commutant of a rationally cyclic subnormal operator. Indeed, if $\mu$ is a measure on the complex plane with compact support $K, R$ is the algebra of rational functions with poles off of $K, R^{2}$ is the $L^{2}(\mu)$ closure of $R$, and $S$ is multiplication by $z$ restricted to $R^{2}$, then the commutant of $S$ is $\left\{M_{\phi}: \phi \in R^{2} \cap L^{\infty}(\mu)\right\}$, where $M_{\phi} f=\phi f$ is the usual multiplication operator. It follows that if $X$ is an operator on $R^{2}$ which commutes with multiplications by elements of $R$, then $X=M_{\phi}$ for some $\phi \in L^{\infty}(\mu)$ with $M_{\phi}\left(R^{2}\right) \subset R^{2}$. So the commutant of $R$ acting on its $L^{2}(\mu)$ closure lifts to the von Neumann algebra $L^{\infty}(\mu)$ acting on $L^{2}(\mu)$. For $\mathcal{T}$ acting on $H^{2}$ we have the following result.

Theorem 1. If $X \in \mathcal{T}^{\prime}$, then there exists $b \in \mathcal{M}$ such that $H^{2}$ is invariant for $R_{b}$, $X=R_{b}$ on $H^{2}$, and $\|X\|=\|b\|$.

Proof. Let $X(1)=h$. First, we will show that $\|r h\|_{2} \leq\|X\|\|r\|_{2}$ for all $r \in \mathcal{M}$. Let $\varepsilon>0$. Then $r^{*} r+\varepsilon 1$ is an invertible positive operator in $\mathcal{M}$. So by [1, Corollary 4.2.4] there is an $a \in \mathcal{A}$ such that $r^{*} r+\varepsilon 1=a^{*} a$. Thus

$$
\begin{aligned}
\left\langle\left(r^{*} r+\varepsilon 1\right) h, h\right\rangle & =\left\langle a^{*} a h, h\right\rangle=\|a h\|_{2}^{2}=\|X a\|_{2}^{2} \\
& \leq\|X\|^{2}\|a\|_{2}^{2}=\|X\|^{2}\left\langle a^{*} a, 1\right\rangle=\|X\|^{2}\left\langle r^{*} r+\varepsilon 1,1\right\rangle .
\end{aligned}
$$

Letting $\varepsilon$ go to 0 , we obtain $\|r h\|_{2}^{2} \leq\|X\|^{2}\|r\|_{2}^{2}$.

Define a map $Y$ via $Y(r)=r h$. Clearly $Y$ extends to a bounded operator on $L^{2}$ which commutes with $\mathcal{L}$, and $\|Y\| \leq\|X\|$. Thus there is a $b \in \mathcal{M}$ such that $Y=R_{b}$. Because $h \in H^{2}, Y$ leaves $H^{2}$ invariant. Obviously $Y$ agrees with $X$ on $H^{2}$, and so $\|b\|=\|Y\|=\|X\|$.

\section{Factorizations, Toeplitz and Hankel operators}

Our next theorem is a useful factorization result for elements of $L^{2}$, which might be expressed as $L^{2}=L^{\infty} H^{2}=H^{2} L^{\infty}$. The proof in the classical case is an 
application of the fact that if $\log |f|$ is integrable for an $L^{2}$ function $f$, then $|f|=|h|$ for some $H^{2}$ function $h$. However, this fact is a consequence of Szegö's Theorem, and it is not yet known if Szegö's Theorem extends to the subdiagonal algebra setting. Nevertheless [10, Proposition 1] is a sufficient substitute. As consequences we obtain some facts about Toeplitz operators, the factorization of elements of $H^{1}$ as products of $H^{2}$ elements (previously obtained by Saito), and Nehari's Theorem.

Theorem 2. For every $\varepsilon>0$ and $z \in L^{2}$, there exist $h_{1}, h_{2} \in H^{2}$ and $v_{1}, v_{2} \in \mathcal{M}$, such that $z=v_{1} h_{1}=h_{2} v_{2},\left\|v_{i}\right\| \leq 1,\left\|h_{i}\right\|_{2}<(1+\varepsilon)\|z\|_{2}$, and $h_{i}^{-1} \in \mathcal{A}$ for $i=1,2$.

Proof. We prove the existence of $v_{1}$ and $h_{1}$. The proof for $v_{2}$ and $h_{2}$ is similar. Choose $\delta<\sqrt{2 \varepsilon+\varepsilon^{2}}\|z\|_{2}$. Consider the positive weak ${ }^{*}$ continuous linear functional $\omega_{\delta 1}+\omega_{z}$ on $\mathcal{R}$. By $\left[7\right.$, Theorem 7.2.3] there is a vector $y \in L^{2}$ such that $\omega_{y}=\omega_{\delta 1}+\omega_{z}$ on $\mathcal{R}$. Clearly, $\omega_{\delta 1} \leq \omega_{y}$ and $\omega_{z} \leq \omega_{y}$ on $\mathcal{R}$. It follows that there exist $r, s \in \mathcal{M}$ such that $\delta 1=r y$ and $z=s y$. Because $\mathcal{M}$ is finite, $\delta^{-1} r$ has an inverse in $L^{2}$.

By [10, Proposition 1] there exist a unitary $u \in \mathcal{M}$ and $g \in \mathcal{A}$ such that $\delta^{-1} r=$ $g u$, and $h=g^{-1} \in H^{2}$. Thus $y=u^{*} h, z=s u^{*} h$, and $\|h\|_{2}=\|y\|_{2}=\sqrt{\delta^{2}+\|z\|_{2}^{2}}<$ $(1+\varepsilon)\|z\|_{2}$. Now let $v_{1}=s u^{*}$ and $h_{1}=h$.

In the classical setting, one has that the map $t \rightarrow T_{t}$ is an isometry on $L^{\infty}$, but the proof relies heavily on the fact that $L^{\infty}$ is commutative. We do not know if this map is isometric in our noncommutative setting. We do have the following partial results, including the fact that the map is very well behaved on $\mathcal{A}$.

Corollary 3. For every $t \in \mathcal{M},\left\|L_{t} P\right\|=\|t\|$.

Proof. We have $\left\|L_{t}\right\|=\|t\|$. So given $\varepsilon>0$, choose $z$ such that $\|z\|_{2}<1$ and $\|t z\|_{2} \geq\|t\|-\varepsilon$. By Theorem 2 we can write $z=h v$ where $v \in \mathcal{M},\|v\| \leq 1$, $h \in H^{2}$, and $\|h\|_{2}<1$. Thus $\|t z\|_{2}=\|t h v\|_{2} \leq\|t h\|_{2}$, so $\|t h\|_{2} \geq\|t\|-\varepsilon$.

Corollary 4. For every $t \in \mathcal{M},\left\|T_{t^{*}}\right\|=\left\|t^{*} t\right\|$.

Proof. We have $\left\|T_{t^{*} t}\right\|=\left\|P L_{t^{*} t} P\right\|=\left\|P L_{t}^{*} L_{t} P\right\|=\left\|L_{t} P\right\|^{2}=\|t\|^{2}=\left\|t^{*} t\right\|$.

Corollary 5. The map $a \rightarrow T_{a}$ is an isometric algebra isomorphism and a weak* homeomorphism of $\mathcal{A}$ onto $\left\{T_{a}: a \in \mathcal{A}\right\}$.

Proof. For $a \in \mathcal{A}, T_{a}=L_{a} P$, so by Corollary 3 the map is an isometry. Because the map $a \rightarrow L_{a}$ is a weak* continuous isomorphism and $H^{2}$ is invariant for $\left\{L_{a}: a \in \mathcal{A}\right\}$, the map $a \rightarrow T_{a}$ is a weak* continuous isomorphism. It follows from $\left[2\right.$, Theorem 1.20] that $\left\{T_{a}: a \in \mathcal{A}\right\}$ is weak* closed and the map is a weak* homeomorphism.

Let $\mathcal{A}_{*}$ denote the space of weak* continuous linear functionals on $\mathcal{A}$. The next result shows that the weak* continuous functionals on the algebra $\left\{T_{a}: a \in \mathcal{A}\right\}$ have a rank one structure. In the language of the theory of dual algebras [2] the algebra has property $\mathbf{A}_{1}(1)$.

Corollary 6. For every $\varepsilon>0$ and for every $\phi \in \mathcal{A}_{*}$, there exist $g, h \in H^{2}$ such that $\phi(a)=\langle a g, h\rangle$ for $a \in \mathcal{A}$, and $\|g\|_{2}\|h\|_{2}<(1+\varepsilon)\|\phi\|$.

Proof. For $\varepsilon>0$, choose $\eta>0$ such that $(1+\eta)^{2}<1+\varepsilon$. There is a functional $\hat{\phi}$ in $\mathcal{M}_{*}$ such that $\hat{\phi}$ extends $\phi$ and $\|\hat{\phi}\|<(1+\eta)\|\phi\|$ by [4, Lemma 2.4]. Because of the duality between $L^{1}$ and $\mathcal{M}$, there is an $f \in L^{1}$ such that $\hat{\phi}(t)=\tau(t f)$ for $t \in \mathcal{M}$ and $\|\hat{\phi}\|=\|f\|_{1}$. So there exist vectors $x, y \in L^{2}$ such that $f=x y$ and 
$\|x\|_{2}\|y\|_{2}=\|f\|_{1}=\|\hat{\phi}\|<(1+\eta)\|\phi\|$. By Theorem $2 x=g v$ with $g \in H^{2}, v \in \mathcal{M}$, $\|v\| \leq 1$, and $\|g\|_{2}<(1+\eta)\|x\|_{2}$. Thus, for all $a \in \mathcal{A}$,

$$
\phi(a)=\left\langle L_{a} g v, y\right\rangle=\left\langle L_{a} g, y v^{*}\right\rangle=\left\langle L_{a} g, P\left(y v^{*}\right)\right\rangle .
$$

Let $h=P\left(y v^{*}\right)$, and the result follows.

We now proceed to establish an analogue of the factorization theorem for $H^{1}$. Recall that any function $f$ in the classical $H^{1}$ space can be written $f=g h$ where $g, h$ are in $H^{2}$ and $\|f\|_{1}=\|g\|_{2}\|h\|_{2}$. The following result was previously obtained by Saito [11, Lemma 5.5], but we include it for completeness.

Corollary 7. For every $\varepsilon>0$ and for every $f \in H^{1}$, there exist $g, h \in H^{2}$ such that $f=g h$ and $\|g\|_{2}\|h\|_{2}<(1+\varepsilon)\|f\|_{1}$. If $f \in H_{0}^{1}$, then $h$ is in $H_{0}^{2}$.

Proof. Because $f \in L^{1}$, there exist vectors $x, y \in L^{2}$ such that $f=x y$ and $\|x\|_{2}\|y\|_{2}=\|f\|_{1}$. By Theorem 2 we can find $g \in H^{2}$ and $v \in \mathcal{M}$ such that $x=g v,\|v\| \leq 1,\|g\|_{2}<(1+\varepsilon)\|x\|_{2}$ and $g^{-1} \in \mathcal{A}$. Now by [10, Lemma 3] $H^{1}=\left\{x \in L^{1}: \tau(x y)=0\right.$ for all $\left.y \in H_{0}^{\infty}\right\}$. Thus, for $a \in \mathcal{A}_{0}$,

$$
\tau(a f)=\tau(a x y)=\tau(\text { agvy })=\left\langle a g,(v y)^{*}\right\rangle .
$$

Let $h=v y$. Then $h^{*} \in\left[\mathcal{A}_{0} g\right]_{2}^{\perp}$. But $\left[\mathcal{A}_{0} g\right]_{2}=\left[\mathcal{A}_{0}\right]_{2}$, because $g^{-1} \in \mathcal{A}$. Thus $h^{*} \in\left[\mathcal{A}_{0}\right]_{2}^{\perp}=\left(H^{2}\right)^{*}$, so $h \in H^{2}$ and $f=x y=g v y=g h$. Finally, $\|g\|_{2}\|h\|_{2}<$ $(1+\varepsilon)\|x\|_{2}\|y\|_{2}=(1+\varepsilon)\|f\|_{1}$.

Note that if $f \in H_{0}^{1}$, then $\tau($ af $)=0$ for all $a \in \mathcal{A}$. So $h^{*} \in\left(H^{2}\right)^{\perp}=\left(H_{0}^{2}\right)^{*}$, and thus $h \in H_{0}^{2}$.

As a consequence of the previous factorization theorem, we can obtain an exact analogue of Nehari's Theorem on the norm of a Hankel operator. In the classical setting, the norm of a Hankel operator $H_{f}$ is given by the distance in $L^{\infty}$ from the symbol $f$ to $H^{\infty}$. This remains true in the noncommutative setting.

Corollary 8. For every $t \in \mathcal{M},\left\|H_{t}\right\|=\operatorname{dist}(t, \mathcal{A})$.

Proof. By [10, Lemma 4] $H_{0}^{1}=\left\{f \in L^{1}: \tau(f y)=0\right.$ for all $\left.y \in \mathcal{A}\right\}$. By Corollary 7 $\left\{f \in H_{0}^{1}:\|f\|_{1}<1\right\}=\left\{g h: g \in H^{2}, h \in H_{0}^{2},\|g\|_{2}\|h\|_{2}<1\right\}$. Because $\mathcal{A}$ is $\mathrm{w}^{*}$-closed,

$$
\begin{aligned}
\operatorname{dist}(t, \mathcal{A}) & =\sup \left\{|\tau(t f)|: f \in L^{1}, \tau(y f)=0 \text { for all } y \in \mathcal{A},\|f\|_{1}<1\right\} \\
& =\sup \left\{|\tau(t f)|: f \in H_{0}^{1},\|f\|_{1}<1\right\} \\
& =\sup \left\{|\tau(t g h)|: g \in H^{2}, h \in H_{0}^{2},\|g\|_{2}\|h\|_{2}<1\right\} \\
& =\sup \left\{\left|\left\langle L_{t} g, h^{*}\right\rangle\right|: g \in H^{2}, h \in H_{0}^{2},\|g\|_{2}\|h\|_{2}<1\right\} \\
& =\left\|H_{t}\right\| .
\end{aligned}
$$

\section{Harmonic COnjugates}

Every harmonic function $u$ on the unit disk has an associated harmonic function $\tilde{u}$, the harmonic conjugate of $u$, such that $u+i \tilde{u}$ is analytic and $\tilde{u}(0)=0$. The map $u \rightarrow \tilde{u}$ is a real linear transformation which is $L^{2}$ bounded. We will establish an analogue of this result by thinking of $\operatorname{Re} \mathcal{A}$, the real parts of the operators in $\mathcal{A}$, as a noncommutative version of the space of bounded harmonic functions. We first construct a real linear map from $\operatorname{Re} \mathcal{A}$ to $\mathcal{A}$ which is the analogue of the Herglotz transform, i.e. integration against the kernel $\left(e^{i \theta}+z\right)\left(e^{i \theta}-z\right)^{-1}$. 
Theorem 9. There is a real linear transformation $T: \operatorname{Re} \mathcal{A} \rightarrow \mathcal{A}$ such that $u=$ $\operatorname{Re}(T u), \Phi(\operatorname{Im} T u)=0$, and $\|T u\|_{2} \leq \sqrt{2}\|u\|_{2}$ for all $u \in \operatorname{Re} \mathcal{A}$. Thus $T$ extends to a bounded operator from $[\operatorname{Re} \mathcal{A}]_{2}$ to $H^{2}$.

Proof. Let $u \in \operatorname{Re} \mathcal{A}$. So $u=\operatorname{Re} g$ for some $g \in \mathcal{A}$. Let $a=g-\frac{1}{2} \Phi\left(g-g^{*}\right)$. Then $a \in \mathcal{A}, u=\operatorname{Re} a$, and $\Phi(\operatorname{Im} a)=0$. Thus there exists an $a \in \mathcal{A}$ with the desired properties. We now show that such an element of $\mathcal{A}$ is unique. Suppose that $u=\operatorname{Re} a=\operatorname{Re} h$ for $a, h \in \mathcal{A}$, and $\Phi(\operatorname{Im} a)=\Phi(\operatorname{Im} h)=0$. Then $a+a^{*}=h+h^{*}$ implies that $a-h=h^{*}-a^{*}$, so $(a-h)^{*}=h-a$. Furthermore, $\Phi(\operatorname{Im} a)=\Phi(\operatorname{Im} h)=0$ implies that $\Phi(a)=\Phi\left(a^{*}\right)$ and $\Phi(h)=\Phi\left(h^{*}\right)$. Consequently, $\Phi(a)=\Phi(h)$, because Re $a=\operatorname{Re} h$. Thus

$$
\Phi\left((a-h)(a-h)^{*}\right)=\Phi((a-h)(h-a))=\Phi(a-h) \Phi(h-a)=0 .
$$

So $a=h$, because $\Phi$ is faithful. Thus we can define $T u=a$, where $a$ is the unique element of $\mathcal{A}$ with $u=\operatorname{Re} a$ and $\Phi(\operatorname{Im} a)=0$. It is easy to see that $T$ is real linear.

We now proceed to establish the inequality $\|T u\|_{2} \leq \sqrt{2}\|u\|_{2}$. Let $T u=d+b$, where $d \in \mathcal{D}, b \in \mathcal{A}_{0}$. Now $\Phi(\operatorname{Im} T u)=0$ and $\Phi(b)=\Phi\left(b^{*}\right)=0$, so we have $d=\Phi(d)=\Phi\left(d^{*}\right)=d^{*}$. Thus

$$
\|T u\|_{2}^{2}=\tau\left((d+b)\left(d+b^{*}\right)\right)=\|d\|_{2}^{2}+\tau\left(d b^{*}\right)+\tau(d b)+\|b\|_{2}^{2}=\|d\|_{2}^{2}+\|b\|_{2}^{2} .
$$

By a simple computation we have

$$
0=\tau\left(b^{2}\right)=\tau\left((\operatorname{Re} b)^{2}\right)-\tau\left((\operatorname{Im} b)^{2}\right)+2 i \tau((\operatorname{Re} b)(\operatorname{Im} b)) .
$$

Note that

$$
\overline{\tau((\operatorname{Re} b)(\operatorname{Im} b))}=\tau((\operatorname{Im} b)(\operatorname{Re} b))=\tau((\operatorname{Re} b)(\operatorname{Im} b)) .
$$

By equating real and imaginary parts we obtain

$$
\|(\operatorname{Re} b)\|_{2}^{2}=\|(\operatorname{Im} b)\|_{2}^{2} \text { and } \tau((\operatorname{Re} b)(\operatorname{Im} b))=0 .
$$

So

$$
\|b\|_{2}^{2}=\|(\operatorname{Re} b)\|_{2}^{2}+\|(\operatorname{Im} b)\|_{2}^{2}=2\|(\operatorname{Re} b)\|_{2}^{2} .
$$

Thus

$$
\|T u\|_{2}^{2}=\|\operatorname{Re} d\|_{2}^{2}+2\|\operatorname{Re} b\|_{2}^{2} .
$$

Now because $b$ is orthogonal to $\mathcal{D}$, we have $\tau((\operatorname{Re} d)(\operatorname{Re} b))=0$. Consequently,

$$
\|T u\|_{2}^{2} \leq 2\left(\|\operatorname{Re} d\|_{2}^{2}+\|\operatorname{Re} b\|_{2}^{2}\right)=2\|\operatorname{Re}(d+b)\|_{2}^{2}=2\|u\|_{2}^{2} .
$$

Corollary 10. For each $u \in[\operatorname{Re} \mathcal{A}]_{2}$ there is a unique $\tilde{u}$ in $[\operatorname{Re} \mathcal{A}]_{2}$ such that $u+i \tilde{u} \in H^{2}$ and $\Phi(\tilde{u})=0$. Furthermore, $\|\tilde{u}\|_{2} \leq \sqrt{2}\|u\|_{2}$.

Proof. Let $\tilde{u}=\operatorname{Im} T u$.

\section{REFERENCES}

1. W. Arveson, Analyticity in operator algebras, Amer. J. Math. 89 (1967), 578-642. MR 36:6946

2. H. Bercovici, C. Foiaş, and C. Pearcy, Dual algebras with applications to invariant subspaces and dilation theory, CBMS Regional Conf. Ser. in Math., no. 56, Amer. Math. Soc., Providence, RI, 1985. MR 87g:47091

3. J. Dixmier, Formes linéaires sur un anneau d'opérateurs, Bull. Soc. Math. France 81 (1953), 9-39. MR 15:539a

4. D. Hadwin and E. Nordgren, Subalgebras of reflexive algebras, J. Operator Theory 7 (1983), 3-23. MR 83f: 47033 
5. Y. Imina and K.-S. Saito, Hankel operators associated with analytic crossed products, Can. Math. Bull. 37 (1994), 75-81. MR 94k:47024

6. N. Kamei, Simply invariant subspace theorems for antisymmetric finite subdiagonal algebras, Tohoku Math. J. 21 (1969), 467-473. MR 41:839

7. R. Kadison and J. Ringrose, Fundamentals of the Theory of Operator Algebras, Academic Press, New York, 1983. MR 85j:46099

8. M. McAsey, P. Muhly and K.-S. Saito, Nonselfadjoint crossed products (invariant subspaces and maximality), Trans. Amer. Math. Soc. 248 (1979), 381-410. MR 80j:46101b

9. E. Nelson, Notes on non-commutative integration, J. Functional Analysis 15 (1974), 103-116. MR 50:8102

10. K.-S. Saito, A note on invariant subspaces for finite maximal subdiagonal algebras, Proc. Amer. Math. Soc. 77 (1979), 348-352. MR 81b:46078

11. _ Toeplitz operators associated with analytic crossed products, Math. Proc. Cambridge Phil. Soc. 108 (1990), 539-549. MR 91m:46109

12. I. Segal, A noncommutative extension of abstract integration, Ann. of Math. 57 (1953), 401457. MR 14:991f

13. T. Yoshino, Subnormal operators with a cyclic vector, Tôhoku Math. J. 21 (1973), 47-55. MR 39:7450

Department of Mathematics, Illinois State University, Normal, Illinois 61790-4520

E-mail address: marsalli@math.ilstu.edu 